\title{
Real world outcomes of ocriplasmin use by members of the British and Eire Association of Vitreoretinal Surgeons
}

RJ Haynes ${ }^{1}$, D Yorston², DAH Laidlaw ${ }^{3}$, J Keller ${ }^{1}$ and DHW Steel ${ }^{4}$

\begin{abstract}
Purpose To describe a survey of the use of ocriplasmin by members of the British and Eire Association of VitreoRetinal Surgeons (BEAVRS) for VitreoMacular Traction (VMT) and Full Thickness Macular Hole (FTMH) and compare it to published MIVI TRUST trial data.

Methods All 173 BEAVRS members were contacted by email in October 2014 requesting data on all cases treated with ocriplasmin up to that date. The total number of cases, FTMH closure rate, VMT release rate and the frequency of adverse events were recorded. Results were compared with trial data.

Results 48 members responded reporting results from 241 eyes. The respective BEAVRS and MIVI TRUST trial closure rates for small FTMHs were 42.1 and $58.3 \%$ $(P=0.09)$ and for medium FTMH 12.7 and $36.7 \%(P=0.01)$. The respective VMT release rates were 34.1 and $37.4 \%(P=N S)$. Retinal detachment was observed in $3.3 \%$ of the BEAVRS cohort compared with $0.4 \%$ in MIVI TRUST. Reduction in visual acuity to $<6 / 60$ was observed in $5.8 \%$ of the BEAVRS cohort and $0.6 \%$ in MIVI TRUST. Other complications not reported in the MIVI TRUST trial included an increase in FTMH basal diameter following unsuccessful ocriplasmin use in $46.9 \%$ of BEAVRS cases and zonular instability at the time of subsequent phacoemulsification in $2.4 \%$. Conclusion Macular hole closure rates were lower in the BEAVRS survey than published in the MIVI TRUST trial data. The incidence of adverse events was greater than previously reported. The reasons for these disparities are unknown but could include positive reporting bias inherent to retrospective surveys, treatment and population differences.
\end{abstract}

Eye (2017) 31, 107-112; doi:10.1038/eye.2016.195; published online 2 September 2016

\section{Introduction}

Ocriplasmin is a first in class agent licensed within Europe in January 2013 for the pharmacological treatment of VitreoMacular Traction (VMT) including that associated with full thickness macular holes (FTMH).

The efficacy and side effect profile of ocriplasmin had been demonstrated in the Microplasmin for Intravitreous InjectionTraction Release without Surgical Treatment (MIVI TRUST) pivotal trials (TG-MV-006 and TG-MV-007) reported in August 2012. ${ }^{1}$ The authors concluded that 'Intravitreal injection of ocriplasmin was superior to injection of placebo in altering the vitreoretinal interface of affected eyes, although it was accompanied by some, mainly transient, ocular adverse events. ${ }^{1}$ Separate analysis of the safety data concluded 'Ocriplasmin seems to be generally safe and well tolerated'.2 A subsequent publication performed a sub group analysis of the MIVI TRUST data in which macular holes were divided into small and medium sized holes, that is those with an internal diameter or $<250$ microns or between 250 and 400 microns, respectively. ${ }^{3}$ Based upon these data ocriplasmin was recommended in October 2013 by the National Institute for Health and Care Excellence (NICE) for use within the NHS in England. Prior to this recommendation the post licensing use of ocriplasmin by BEAVRS members was limited to the minority private healthcare sector.

Anecdotal reports of adverse events combined with early reports in the literature of previously unreported side effects, such as enlargement of the macular hole, zonular instability, and localised non-rhegmatogenous retinal detachment ${ }^{4,5,6}$ prompted this survey of all BEAVRS members. The aim of this study was
${ }^{1}$ Bristol Eye Hospital, Bristol, UK

${ }^{2}$ BEAVRS Research Lead \& Gartnavel Hospital Glasgow, Glasgow, UK

${ }^{3}$ BEAVRS President \& St Thomas' Hospital, London and The Maidstone Hospital, Kent, UK

\footnotetext{
${ }^{4}$ Sunderland Eye Infirmary, Sunderland, UK and Institute of Genetic Medicine, Newcastle University, Newcastle Upon Tyne, UK

Correspondence: RJ Haynes, Bristol Eye Hospital, University Hospitals Bristol NHS Trust, Lower Maudlin Street, Bristol BS1 2LX, UK Tel: +44 (0)117 342 1401; Fax: +44 (0)1173423402. E-mail: Richard.Haynes@ uhbristol.nhs.uk
}

Received: 8 February 2016 Accepted in revised form: 11 July 2016 Published online: 2 September 2016 
to ascertain the effectiveness of ocriplasmin and the incidence of adverse events in normal clinical practice. To date no systematic national post marketing surveillance studies have been reported.

\section{Materials and methods}

All 173 BEAVRS members (including 120 consultants) with a registered email address were contacted electronically in October 2014. They were asked to report all cases of VMT with or without full thickness macular holes (FTMH) that they had treated with ocriplasmin. Members were requested to complete a simple spreadsheet (Table 1). The data set included the total number of cases they had treated with ocriplasmin, the case mix divided into small FTMH ( $<250$ microns), medium FTMH ( $>250$ and $<400$ microns) or pure VMT without FTMH, and the proportion with a successful anatomical outcome (ie, closure of FTMH or release of VMT). Adverse events surveyed included reduction in visual acuity to less than 6/60 Snellen, severe photopsia, photophobia, dyschromatopsia*, pain, retinal tear and/or retinal detachment, changes in the outer segment ellipsoid layer on optical coherence tomography*, electroretinogram changes*, increase in measured macular hole basal diameter*, zonular instability during subsequent cataract surgery* and subsequent failure of macular hole surgery*. Items marked with an asterisk were not reported in the MIVI TRUST publications.

A second email was sent in January 2015 to collect additional cases and remind contributors of the importance of including data on all cases treated, without selection. $\chi^{2}$-tests for differences in proportion were employed.

Table 1 BEAVRS survey spreadsheet

Number of cases

Total Ocriplasmin cases treated

Small MH $(<250 \mu)$

Medium MH $(250-400 \mu)$

Pure VMT (no MH)

Closure of Small MH

Closure of Medium MH

Release of Pure VMT

Profound drop in VA $(<6 / 60)$

Severe photopsia

Photophobia

Dyschromatopsia

Pain

Loss of ellipsoid layer on OCT

Increased $\mathrm{MH}$ basal diameter

ERG changes

Retinal Tear

Retinal Detachment

Evidence of Zonular instability

Subsequent $\mathrm{MH}$ surgery failure

\section{Results}

Forty-one members responded to the survey that they had used ocriplasmin, in addition, seven members responded that they had not used ocriplasmin (even though this was not an option in the survey) representing an overall response rate from consultant members of $40 \%$. In the United Kingdom non-consultants would be unlikely to use ocriplasmin without consultant supervision, in addition, at the time of surveying many consultant BEAVRS had not used ocriplasmin but the exact number is unknown as the survey did not ask this. However, at the BEAVRS meeting in 2014 there was an 'ask the audience' question about the preferred treatment option for a patient with a small FTMH-66\% voted surgery, $8 \%$ voted ocriplasmin and $26 \%$ voted observation, suggesting a low usage of ocriplasmin by BEAVRS at the time, consistent with the survey results. A total of 241 eyes were reported to have been injected with ocriplasmin. The distribution of cases is shown in Table 2. The respective BEAVRS and MIVI TRUST closure rates of small FTMHs were 42.1 and $58.3 \%(P=0.09)$ and for medium sized FTMH 12.7 and $36.7 \%(P=0.01)$. The outcomes for resolution of VMT were similar to the MIVI TRUST findings with successful resolution of VMT in 34.1 and $37.4 \%$, respectively. A summary of the BEAVRS results with the percentages of each outcome relative to the total number of BEAVRS cases and the equivalent MIVI TRUST percentage results for comparison are shown in Table 2. Profound drop in vision to $<6 / 60$ was reported in $5.8 \%$ of cases, with one case falling temporarily to perception of light only (PL) for $24 \mathrm{~h}$. $11.6 \%$ of patients in the BEAVRS cohort experienced severe photopsia. Photopsia was described by some patients as like a 'snow storm' or a 'poorly tuned TV' and by others as like a 'New Year's Eve firework display'. Dyschromatopsia (mainly yellowing or dimming of vision) was reported in $6.2 \%$ of BEAVRS ocriplasmin cases. Changes in the outer segment ellipsoid layer on OCT were identified in $10.4 \%$. Ocriplasmin treatment of FTMH failed in 81/112 cases (72\%); in these failed cases widening of the macular hole basal diameter on OCT scanning was reported in $46.9 \%$ (Figure 1). In one case sub-retinal fluid/lucency was identified in a region distal to the $\mathrm{MH}$ around the optic nerve, including on the nasal side of the nerve (Figure 2).

ERG changes were reported in six cases; however, ERGs were only performed in some of the cases that reported marked visual loss. The reported ERG changes consisted of a marked reduction in a and b wave amplitudes and loss of oscillatory potentials suggesting widespread retinal dysfunction in all cases reported (Figure 3). We have no detailed data on how rapidly the ERG changes developed or the speed of recovery, other 
Table 2 Results of BEAVRS survey with equivalent percentages from MIVI TRUST trials for comparison

\begin{tabular}{lccc}
\hline & BEAVRS totals & BEAVRS \% (95\% CI) & MIVI TRUST \% \\
\hline Total Ocriplasmin cases treated & 241 & & 464 \\
Small MH $(<250 \mu)$ & 57 & 23.7 & 10.3 \\
Medium MH $(250-400 \mu)$ & 55 & 53.5 & 7.2 \\
Pure VMT (no FTMH) & 129 & & 77.2 \\
& & $42.1(29.4-55.9)$ & 58.3 \\
Closure of Small MH & 24 & $12.7(5.7-25.1)$ & $36.8(P=0.01)$ \\
Closure of Medium MH & 7 & $34.1(24.7-40.1)$ & 37.4 \\
Release of Pure VMT & 44 & $5.8(3.3-9.6)$ & 0.6 \\
Profound drop in VA $(<6 / 60)$ & 14 & $11.6(7.8-16.2)$ & 11.8 \\
Severe photopsia & 28 & $2.9(1.3-6.0)$ & 0.0 \\
Photophobia & 7 & $6.2(3.6-10.1)$ & 1.6 \\
Dyschromatopsia & 15 & 0 & 13.5 \\
Pain & 0 & 10.4 & NR \\
Loss of ellipsoid layer on OCT & 25 & 46 & NR \\
Increased in FTMH basal diameter (in failed) & $38(n=81)$ & 2.5 & NR \\
ERG changes & 6 & $3.3(1.5-6.5)$ & 0.4 \\
Retinal Tear & 8 & $3.3(1.5-6.5)$ & 0.4 \\
Retinal Detachment & 8 & 2.4 & NR \\
Evidence of Zonular instability & 4 & 1.2 & NR \\
Subsequent MH surgery failure & 1 & & \\
\hline
\end{tabular}

Abbreviations: BEAVRS, British and Eire Association of Vitreoretinal Surgeons; ERG, electroretinogram; FTMH, full thickness macular hole; MH, macular hole; MIVI TRUST, Microplasmin for Intravitreous Injection-Traction Release without Surgical Treatment; $n$, denominator number of patients; NR, not recorded; $P, \chi^{2}$-test $P$-values; $\mathrm{OCT}$, optical coherence tomography; VA, Snellen visual acuity; VMT, vitreomacular traction; $\mu$, micrometres; $95 \% \mathrm{CI}, 95 \%$ confidence intervals.

\section{Before ocriplasmin}

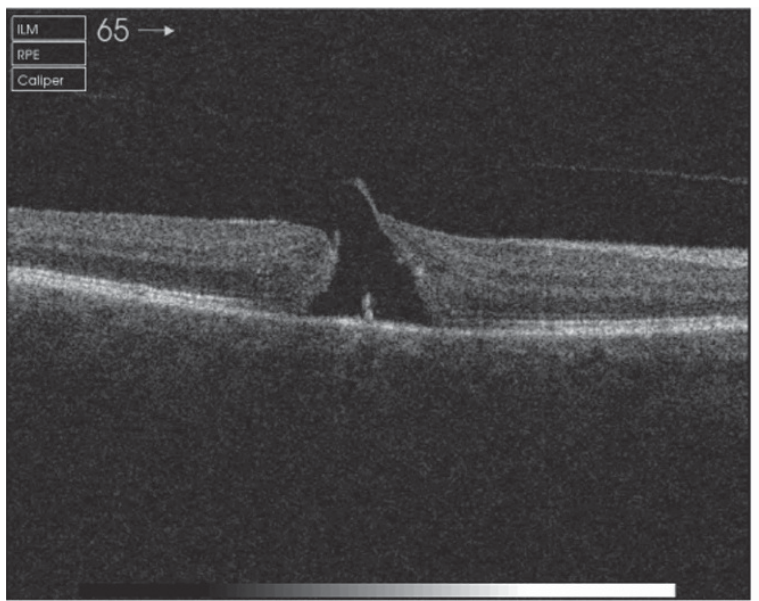

After ocriplasmin.

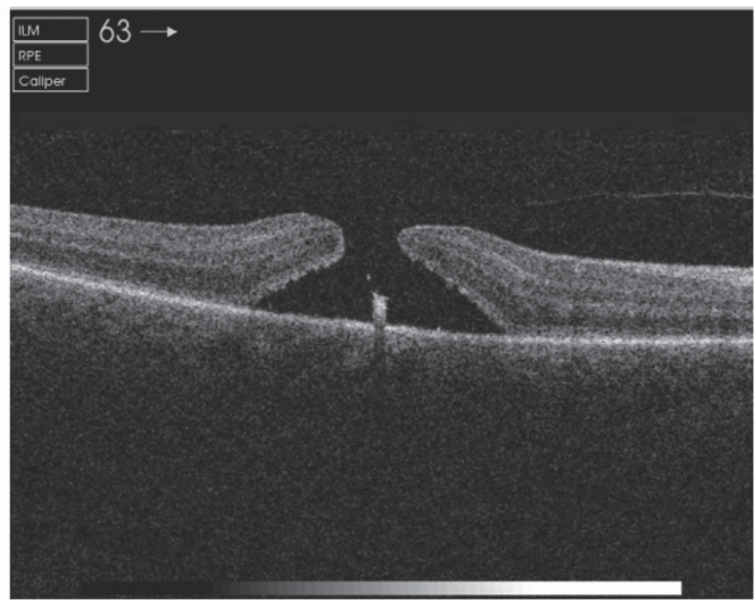

Figure 1 Widening of the MH basal diameter following ocriplasmin.

than to say that the effect was prominent enough to be detected between 2 and 3 weeks after the ocriplasmin injection and in a few cases a relative suppression of the waveforms (compared with the untreated eye) was still present as far as 12 months after the ocriplasmin injection.

Zonular dialysis was reported in four cases following ocriplasmin. Two cases occurred at the time of combined phacoemulsification+lens implant+pars plana vitrectomy surgery for macular holes that had failed to respond to ocriplasmin treatment. The vitrectomy and phacoemulsification surgery in each case was reported as uneventful; however, partial zonular dialysis occurred in one case during intraocular lens injection into the capsular bag. In the second case complete zonular rupture occurred during lens injection necessitating complete removal of the lens and capsular bag and insertion of an iris clip lens. The third case occurred during cataract surgery performed several months after a vitrectomy and gas repair of a retinal detachment subsequent to ocriplasmin injection. In this case the zonules appeared to be absent in one quadrant at the beginning of the phacoemulsification. Phacodonesis had not been noted 

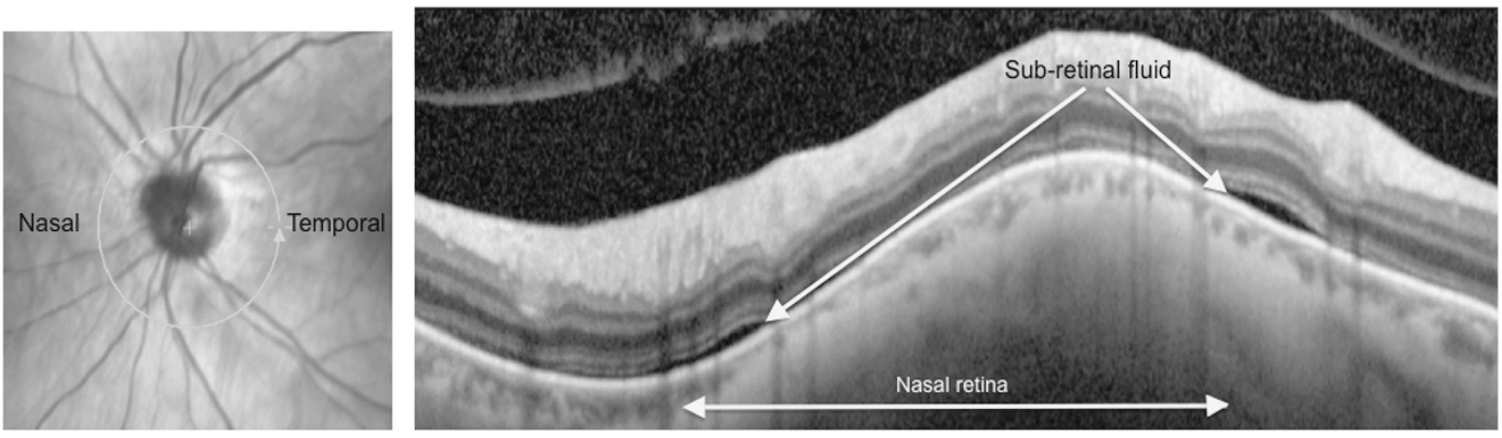

Figure 2 Sub-retinal fluid around the optic nerve, including on the nasal side of the nerve following ocriplasmin.

\section{DARK ADAPTED RESPONSES}

\section{ROD RESPONSE}

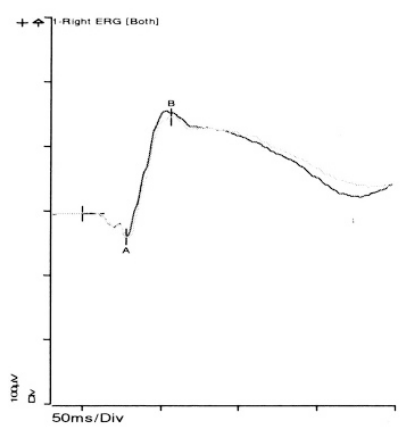

MAXIMAL RESPONSE

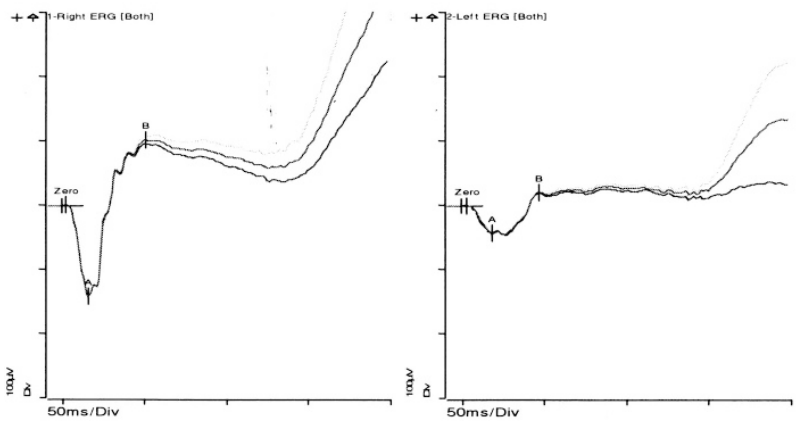

\section{HIGH INTENSITY ROD RESPONSE}

$+$
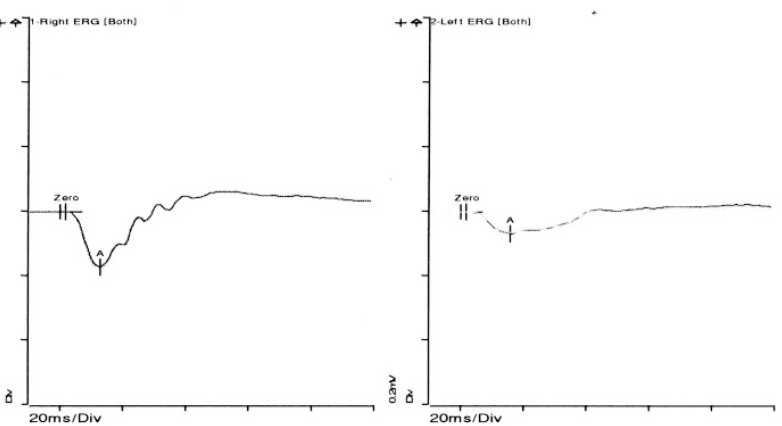

OSCILLATORY POTENTIALS

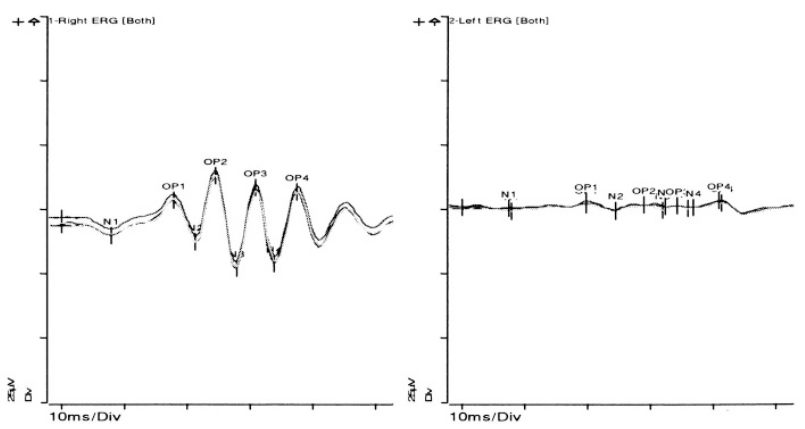

Figure 3 ERG changes following ocriplasmin showing a marked reduction in dark adapted A and B wave amplitudes and loss of oscillatory potentials. The left eye was treated with ocriplasmin.

preoperatively. The fourth case occurred during the irrigation/aspiration step of cataract surgery performed 12 months after vitrectomy performed for persistent VMT following ocriplasmin.

Retinal detachment was found in 8 out of $241(3.3 \%)$ cases reported. There were no post-injection retinal tears that did not progress to retinal detachment.

\section{Discussion}

The reported efficacy from the BEAVRS cohort was similar to that in the MIVI TRUST phase 3 trials for resolution of VMT. However, the BEAVRS rate of closure of medium sized macular holes was significantly lower than published data $(P=0.01)$. Interestingly only 19 eyes in the MIVI TRUST trials had medium sized FTMHs ${ }^{3}$ as compared with the larger sample size of 55 in the BEAVRS cohort.

There was a higher than expected incidence of ocriplasmin related side effects reported in the BEAVRS survey. These include changes in the ellipsoid zone, widening of the basal diameter of macular holes in which ocriplasmin treatment was unsuccessful, ERG abnormalities, the observation of localised non- 
rhegmatogenous retinal detachment, and the development of a rhegmatogenous retinal detachment in all cases where a post ocriplasmin retinal tear occurred. Grouped together these observations indicate ocriplasmin has a more widespread action than the target vitreoretinal interface, and are suggestive of enzyme action both within the retina and the sub-retinal space, rather than just at the target vitreoretinal interface. Muller glial cell disruption and subsequent recovery (being the only cell type capable of regeneration within the retina) has been proposed as the basis for the electrophysiological changes, ${ }^{8}$ as the ERG has been reported to recover over a period of months following ocriplasmin injection. Laminin and fibronectin are found in the interphotoreceptor matrix and contribute to retinal adhesion. It is possible that ocriplasmin gains access to the sub-retinal space via the macular hole; however, with a molecular weight of $27.2 \mathrm{kDa}$, it may be a small enough molecule to penetrate through the retina. ${ }^{9}$ This trans-retinal route would explain the observation of sub-retinal fluid around the optic nerve head including on the nasal side of the nerve. ${ }^{7}$ Further evidence for an adverse effect on retinal adhesion is that all eight of the BEAVRS cases with a retinal tear went on to develop a retinal detachment, whereas natural history studies of spontaneous posterior vitreous detachment suggest that only $30-50 \%$ of tears progress to retinal detachment. ${ }^{10-12}$

The $46.9 \%$ incidence of widening of the FTMH basal diameter in failed ocriplasmin cases could have implications for visual outcomes, as increased basal diameter has previously been found to predict worse post-operative vision; ${ }^{13}$ however, this was not found in the MIVI TRUST trials where the acuity improvement in failed ocriplasmin eyes was the same as in placebo treated eyes. ${ }^{14,15}$ There is uncertainty as to the cause of the observed widened basal diameter. It has been hypothesised that it may relate to the effect of increased vitreous traction on the hole during vitreolysis. ${ }^{16}$

However, the extent of widening does not vary with the degree of VMT release, which might have been expected if this was the cause and furthermore no increase in base diameter has been observed on intraoperative OCTs performed during surgery with either posterior hyaloid face separation or ILM peeling. ${ }^{17,18}$ As with the other outer retinal changes associated with ocriplasmin, widening of the basal diameter is likely to be due to the proteolytic action of ocriplasmin on laminin in the interphotoreceptor matrix, which is partly responsible for adhesion of the retina to the RPE.

The observed $3.3 \%$ rate of retinal detachment in the BEAVRS cohort is much higher than the $0.4 \%$ reported in the MIVI TRUST report. A contributing factor to this discrepancy may be that the MIVI TRUST phase 3 trials of ocriplasmin excluded patients with high myopia, one of the BEAVRS retinal detachments observed was in a patient with -9 dioptres of myopia. Exclusion of this individual results in a $2.9 \%$ rate of retinal detachment within the BEAVRS cohort compared with $0.4 \%$ in the published trials. There are a number of other possible reasons for differences between the BEAVRS and MIVI Trust data. The BEAVRS survey was retrospective and only $40 \%$ of consultant members contributed. It was performed 20 months after European licensing and one year after recommendation by NICE for use on the NHS in England. The disparity in the reported results may arise from positive reporting bias by members who were disappointed with their results. ${ }^{19}$ Similarly, the respondents may have under-reported the total number of cases that they had performed. Either error would lead to a higher proportion of complications or treatment failures. Attempts were made to overcome these biases by reminding members to report all cases to maximise the denominator. The BEAVRS results may also be subject to negative reporting bias with underestimation of the incidence of adverse events. Not all patients will have reported their symptoms or had them systematically recorded in the case notes. In addition, only a few patients underwent ERG tests and not all clinicians would have rigorously measured $\mathrm{MH}$ basal diameter. This is however the largest reported cohort to date reporting real world outcomes of ocriplasmin.

The reduced rate of closure in medium sized FTMH seen in the BEAVRS cohort could be explained by larger diameters within the BEAVRS medium hole size group compared with the MIVI TRUST cohort, but data to support or refute this are not available in either series. Unlike the MIVI TRUST trials, no eyes had ERMs pre-injection, as UK NICE guidelines exclude patients from treatment with ocriplasmin if there is any ERM present.

Differences in injection technique could be important. Ocriplasmin is stored at $-25^{\circ} \mathrm{C}$ in an acidic $\mathrm{pH}$. Once warmed and diluted with non-buffered saline, it starts to autolyse and has a rapid degradation profile within the eye. Differences in injection timing, and location within the vitreous cavity, could lead to variations in outcome. It is recommended that ocriplasmin is given with a $13 \mathrm{~mm} 30$ gauge needle fully entered into the eye, but we have no knowledge of whether this was standard practice. Too anterior a location may reduce efficacy at the vitreo-retinal interface, but increase the risk of zonule weakness. Zonule dehiscence was not reported in the MIVI TRUST data, and may be underestimated as zonular instability may not become apparent until cataract surgery is performed in larger numbers in the future. Other possible explanations for the variation in efficacy and side effects observed may exist in the biological action of ocriplasmin on the multiple forms of laminin present in the human retina. ${ }^{20}$ These splice variants may 
be subject to genetic variation within and between different populations.

In conclusion, in this retrospective email survey of BEAVRS members, we found both a higher incidence of adverse events after ocriplasmin, and lower success rates for macular hole closure, than described in the phase 3 trials. Further real world studies are recommended to further clarify the safety and efficacy of ocriplasmin.

\section{Summary}

What was known before

- Ocriplasmin was previously reported to have a low rate of serious complications such as retinal detachment $(0.4 \%)$.

- Medium sized macular hole closure rates were reported to be $36.7 \%$ with ocriplasmin.

What this study adds

- The frequency and severity of side effects were found to be greater eg. retinal detachment rate of 3.3\% (8 times higher than previously published).

- Macular hole closure rates for medium sized holes were found to be significantly lower than previously published $(12.7 \%)$.

\section{Conflict of interest}

RJ Haynes: Alcon ocriplasmin advisory board member. D Steel: Alcon Consultancy, Research funding. DAH Laidlaw: Alcon ocriplasmin advisory board member. This work received no support in the form of grants, equipment or drugs.

\section{Acknowledgements}

To members of the British and Eire Association of Vitreoretinal Surgeons who contributed their ocriplasmin data including: J Bainbridge, M Bindra, A Callear, A Caswell, S Charles, A Fitt, I Fawcett, T Fallon, C Goldsmith, R Haynes, R Hillier, M La Cour, A Laidlaw, S Lesnik-Oberstein, S Lett, L Membrey, M Muqit, R Newsom, J Park, N Patton, N Price, R Rahman, J Sharkey, J Smith, D Steel, V Tanner, D Vayalambrone, S Murjaneh, K Xue, R Zakir \& H Zambarackji.

\section{References}

1 Stalmans P, Benz MS, Gandorfer A, Kampik A, Girach A, Pakola $\mathrm{S}$ et al. Enzymatic vitreolysis with ocriplasmin for vitreomacular traction and macular holes. N Engl J Med 2012; 367(7): 606-615.

2 Kaiser PK, Kampik A, Kuppermann BD, Girach A, Rizzo S, Sergott RC. Safety profile of ocriplasmin for the pharmacologic treatment of symptomatic vitreomacular adhesion/traction. Retina 35(6): 1111-1127.

3 Haller JA, Stalmans P, Benz MS, Gandorfer A, Pakola SJ, Girach A et al. MIVI-TRUST Study Group. Efficacy of intravitreal ocriplasmin for treatment of vitreomacular adhesion: subgroup analyses from two randomized trials. Ophthalmology 2015; 122(1): 117-122.

4 Tibbetts MD, Reichel E, Witkin AJ. Vision loss after intravitreal ocriplasmin: correlation of spectral -domain optical coherance tomography and electroretinography. JAMA Ophthalmol 2014; 132(4): 487-490.

5 Fahim AT, Khan NW, Johnson MW. Acute panretinal structural and functional abnormalities after intravitreous ocriplasmin injection. JAMA Ophthalmol 2014; 132(4): 484-486.

6 Keller J, Haynes RJ. Zonular dehiscence at the time of combined vitrectomy and cataract surgery after intravitreal ocriplasmin injection. JAMA Ophthalmol 2015; 133(9): 1091-1092.

7 Hager A, Seibel I, Riechardt A, Rehak M, Joussen AM. Does ocriplasmin affect the RPE-photoreceptor adhesion in macular holes? Br J Ophthalmol 2015; 99(5): 635-638.

8 Qiu TG. Ocriplasmin and Muller Glia. Adv Ophthalmol Vis Syst 2014; 1(1): 2.

9 Jackson TL, Antcliff RJ, Hillenkamp J, Marshall J. Human retinal molecular weight exclusion limit and estimate of species variation. Invest Ophthalmol Vis Sci 2003; 44(5): 2141-2146.

10 Colyear BH, Pischel DK. Clinical tears in the retina without detachment. Am J Ophthalmol 1956; 41: 773-792.

11 Linder B. Acute posterior vitreous detachment and its retinal complications. Acta Ophthalmol 1966; 87: 1-108.

12 Davis MD. Natural history of retinal breaks without detachment. Arch Ophthalmol 1974; 92: 183-194.

13 Wakely L, Rahman R, Stephenson J. A comparison of several methods of macular hole measurement using optical coherence tomography, and their value in predicting anatomical and visual outcomes. Br J Ophthalmol 2012; 96(7): 1003-1007.

14 Dugel P, Regillo C, Eliott D. Characterization of anatomic and visual function outcomes in patients with full-thickness macular hole in ocriplasmin phase 3 trials. Am J Ophthalmol 2015; 160(1): 94-99.

15 Greven MA, Garg S, Chiu B, Shah SP, Wolfe J, Fine HF et al. Vitrectomy After Ocriplasmin for VitreOmacular Adhesion Or Macular Hole (VAVOOM) Study. Br J Ophthalmol 2016; 100(9): 1211-1215.

16 Warrow DJ, Lai MM, Patel A, Raevis J, Berinstein DM. Treatment outcomes and spectral-domain optical coherence tomography findings of eyes with symptomatic vitreomacular adhesion treated with intravitreal ocriplasmin. Am J Ophthalmol 2015; 159(1): 20-30.

17 Steel DH, Sandinha MT, White K. The plane of vitreoretinal separation and results of vitrectomy surgery in patients given ocriplasmin for idiopathic macular hole. Invest Ophthalmol Vis Sci 2015; 56(6): 4038-4044.

18 Tadayoni R Foveal Detachment after Idiopathic Macular Hole Surgery. Presented at Gonin society meeting 2014.

19 Delgado-Rodríguez M, Llorca J. Bias. J Epidemiol Community Health 2004; 58(8): 635-641.

20 Colognato H, Yurchenco P. Form and function: the laminin family of heterotrimers. Dev Dyn 2000; 218(2): 213-234. 